RAYO CATALINA - Género performativo y crítica de una estructura capitalista: la obra de Gabriela Rivera, Bestiario (2012-2018)

\title{
Género performativo y crítica de una estructura capitalista: la obra de Gabriela Rivera, Bestiario (2012-2018)
}

Performative and critical genre of a capitalist structure: the work of Gabriela Rivera, Bestiario (2012-2018)

Catalina Rayo ${ }^{1}$

UNIVERSIDAD DE CHILE

Resumen. La obra de la artista Gabriela Rivera, Bestiario, es una expresión grotesca, entre máscaras de carne y performance. En cierta medida, alude a los trabajos de arte de los años setenta sobre fluidos, sangre y lo animal de artistas de esa época, como Ana Mendieta, Cecilia Vicuña, Carol Schneemann y Vito Acconci. La obra trata una materialidad orgánica, con el consumo de carne como parte de una expresión performativa del género. El artículo a continuación intenta dar luces sobre cómo podemos relacionar el capital y el género a partir de una mirada feminista que evidencia lo opresivo.

Palabras clave. Feminismo; Arte; Capitalismo; Géneros performáticos.

\begin{abstract}
The artist Gabriela Rivera's piece Bestiary expresses the grotesque with a play between meat masks and performance. In a certain way, it alludes to art of the 70s which dealt in fluid, blood, and the animal from artists such as Ana Mendieta Cecilia Vicuña, Carol Schneemann and Vito Acconci. Rivera's work deals in an organic materiality, drawing on the consumption of meat to conceptually evoke performative expressions of gender. This paper will attempt to illuminate relationships between capital and gender using a feminist reading of opression
\end{abstract}

Keywords. Feminism; Art; Capitalism; Gendered performances.

${ }^{1}$ Licenciada en Artes de la Pontificia Universidad Católica; MA en Estudios de Cine de la Columbia University, Nueva York, y Magíster en Periodismo Digital por la Universidad de Santiago. Mail:catalina.rayo@uchile.cl 


\section{Introducción: la nueva ola feminista chilena y el impacto que ha tenido en la producción artística}

Lo orgánico, lo animal, lo biológico, lo femenino, lo natural y lo abyecto; las máscaras hechas de carne en la obra de Gabriela Rivera, Bestiario (2012-2018), revelan muchas capas sobre cómo la sociedad se ha enfrentado con el feminismo, especialmente durante 2018, cuando el país fue habitado por un activismo político feminista (momento que se conocerá como Mayo Feminista). En Chile, "la nueva ola feminista" ha impactado fuertemente en la educación superior y en el activismo político; y varias investigadoras han puestos sus enfoques en los trabajos de Julieta Kirkwood, fundadora de los movimientos feministas en la década de 1980, para poder explicar el contexto chileno.

Alejandra Castillo, por ejemplo, en su libro Julieta Kirkwood: Políticas del nombre propio, indica la urgencia intrínseca de citarla si vamos a hablar sobre feminismo (15). Argumenta que la icónica frase "el feminismo soy yo" de Kirkwood alude a un lugar de identificación ficcional: "A la pregunta ¿qué es feminismo? Solo una leve respuesta: "yo", intento de inscribir el cuerpo y la mujer, en tanto la diferencia, en la trama social" (21). Esto indica y refiere a un punto de fijación. En su artículo "De la revuelta feminista, la historia y Julieta Kirkwood", Castillo apela a las políticas paritarias dentro de las universidades y a la inclusión de líneas de trabajo e investigación feminista (36), argumentando que es necesario mirar la historia bajo una 'operación feminista' (en palabras de Kirkwood), de manera descentralizada (38). En esta misma línea, Patricia Pinto aboga por la importancia de los escritos de Kirkwood cuando analiza una de sus frases que alude a una mirada historiográfica vinculada al género femenino: "De ahí el empeño feminista de Julieta por hacer visible la invisible historia de las mujeres" (59).

¿Pero, por qué mirar hacia atrás y enfocarnos en una socióloga de los años ochenta para hablar del contexto de 2018? ¿Y cómo este se vincula con Bestiario? Dichas investigadoras han puesto especial foco en el libro Feminarios de Kirkwood, el cual da las directrices para una propuesta feminista en la educación (recordemos que uno de los mayores petitorios feministas en 2018 fue el de una educación no sexista). Además, en relación con el contexto del presente, esta obra nos entrega un capítulo en el que se propone una respuesta al sistema patriarcal a través del feminismo y la rebeldía. En él se plantea la manera en que se entiende el patriarcado, postulando que una de sus formas de dominio es a través del conocimiento. Este está sometido por una autoridad, un "dominus" (48), que "no admite conocimientos que puedan poner en cuestión el orden posible (ideado y explicado por él) y, menos aún admite aquellos conocimientos (forma y contenido) que se atreven a postular ordenamientos teóricos alternativos” (44). Bajo esta lógica, Kirkwood admite que el conocimiento social, histórico y político postula un solo camino posible del conocer, donde no puede ser desafiado ni puesto en duda, bajo los parámetros que él mismo ha elaborado. Frente a esta lógica patriarcal, Kirkwood responde:

Es precisamente en este ámbito-mundo en donde aparecen (o reaparecen) las demandas y la reflexión feminista. Aquí es donde el pensamiento feminista se está 
RAYO CATALINA - Género performativo y crítica de una estructura capitalista: la obra de Gabriela Rivera, Bestiario (2012-2018)

realizando. De allí, entonces, su imagen y contenido de contra-cultura, de contra-

dominio y de contra-lenguaje y también de contra-poder (45).

Resulta interesante que la postura de Kirkwood sea mirar el feminismo como un 'contrapoder' y/o un 'contra-lenguaje', pues estas mismas aristas son las que entran en juego en el trabajo de Gabriela Rivera. Bestiario pone en evidencia la violencia del lenguaje cotidiano, lenguaje que tiene sus raíces en un conocimiento político-cultural patriarcal. Rivera, con su obra, consigue evidenciar una mirada fémina (desde 'el-otro'), históricamente no-visible, para entender una postura de contrapoder sobre la cotidianidad del lenguaje. Por ende, la obra se contextualiza en los años previos y de revolución feminista de 2018, cuando los pensamientos y la postura de Kirkwood han retomado relevancia. Dicha revolución (rebeldía) parte de jóvenes que levantan "la bandera de la rebelión y logran que medio Chile se declare "feminista" (Valdés 168). La "nueva ola feminista" llama a 'una rebeldía', a una "superación del patriarcado" (Kirkwood 51), y parte de esa rebeldía se encuentra en la obra de Rivera, una obra que habla a través de lo fémino, de lo invisible, desde la mirada del otro, de lo abyecto.

¿Y de qué se trata la obra? Según la propia $\operatorname{artista}^{2}$, Bestiario es un trabajo de varios años (declarado no terminado), expresado tanto en fotografías de gran formato como en video performance. La obra, hasta 2017, recopila un total de 8 máscaras de carne, elaboradas mediante un proceso de taxidermia, en el que se disecan los animales para conservar su apariencia viva y asociar así a cada animal con consideraciones negativas sobre la figura de la mujer: yegua, zorra, perra, víbora, mosca muerta, arpía, pájara, cerda. El objetivo es evidenciar la violencia de género en la denigración de la cotidianeidad de la palabra.

Bestiario destaca no solo porque habla de un tema que hoy en día es foco de atención, sino por la forma en que lo plantea. Rivera apunta a varias aristas dentro de lo que se entiende por 'femenino'. Una de ellas es develada por el texto curatorial que escriben Mónica y Stella Salinero para la galería Bech y que fue publicado en la revista Atlas, lo cual da indicios para la interpretación de la obra mediante la ritualidad de la crianza:

[...] las escenas nos muestran a una mujer joven, otra madura y una niña, las que son la artista, su madre y su hija: un clan unido por la matrilinealidad. La atmósfera completa resalta el sentido abyecto y oscuro que contienen las relaciones entre abuela, madre e hija/nieta, que cosen sus carnes crudas y se dan forma entre ellas, significadas por los hilos, las agujas y el acto de coserse una a la otra.

\footnotetext{
${ }^{2}$ Extraído de la entrevista que Verónica Sánchez, de Foto-féminas, le realizó a la artista en noviembre de 2017. Recuperado de la web oficial de Foto-féminas: http://gabrielarivera.blogspot.com/p/publicaciones.html.
} 
Efectivamente, Rivera comparte, en la entrevista que da en Foto-féminas, que ha sido influenciada por la labor de su madre y abuela en el tema de la costura. A su vez, tal conexión con la crianza y la narrativa de vida le permite develar otros aspectos de análisis crítico relacionados con el consumo de carne y la performance.

Respecto al primero, las máscaras de Rivera no están hechas de cualquier tipo de carne, sino de los desechos del producto animal que está en venta para el consumo humano (es decir, la piel de la carne, la grasa, etc.). A este acto nos aproximamos mediante los estudios sobre ecofeminismo y capitalismo, puesto que el desecho, incluso originado desde un producto capitalizado (con precio y valor), es reutilizado por la artista como materialidad de vestimenta. Lo segundo alude a un traje que Rivera usa en un acto performatico para vestir la palabra. Ella viste la máscara de carne, en cuanto desecho del capitalismo, como también viste e inscribe en su cuerpo desnudo los diferentes nombres otorgados a la mujer (yegua, zorra, perra, etc.).

La obra de Rivera apunta claramente a la violencia cotidiana que reciben las mujeres a través del lenguaje, pero la manera en que lo expresa es interesante por dos aspectos básicos. El primero, y el más obvio, tiene que ver con la materialidad de la obra en sí misma. El segundo, con el acto performativo que Rivera aplica a la interpretación de estas máscaras. Dichos aspectos otorgan un significado a la obra de la artista y a cómo nosotros, como espectadores, podemos entenderla e interpretarla, además de que establecen conexiones con el acontecer local y la manera en que Chile ha manejado la nueva ola feminista de los últimos años.

\section{Consumo carnívoro y masculino, desecho grotesco y femenino}

Con respecto a la materialidad, Rivera no solo no utiliza cualquier tipo de 'carne' para crear estas máscaras, sino que recicla residuos de carne (pieles, cueros, vísceras y entrañas) de consumo diario (pollo, vacuno, cerdo y pescado). La autora prepara la carne mediante técnicas de taxidermia para, posteriormente, poder coserla y crear una máscara, en un proceso que toma muchos meses, según ella misma explica ${ }^{3}$. Una vez que la máscara está lista, es la misma creadora quien la viste y hace una serie de registros fotográficos con ella. Ahora bien, la relación y la lógica de la obra cobra sentido cuando entendemos que el consumo de carne de animal es posible a través de una transacción económica, pero que, al mismo tiempo, no solo 'se consume carne' sino que se viste con esta carne consumida, relación que transforma a la artista en lo que la carne simboliza. Este vínculo se explica a través del encuentro íntimo al que es sometido un cuerpo y su performatividad a través de un proceso de valorización. Por ende, la artista viste un desecho de carne adquirida económicamente, lo que la convierte, a su vez, en 'consumo': mujer es animal y, como animal, puede ser consumida.

Bajo esta lógica, la mujer pasa de ser individua autónoma a ser objeto de consumo. Ello nos permite entrever las distintas implicancias que esto conlleva. Si ella es objeto de consumo, ¿para qué se consume? La analogía de la obra nos hace pensar en el tema de la reproductibilidad. En congruencia, no podemos dejar de señalar la importancia de este tema (y, para el caso, de las relaciones heterosexuales) en el sistema capitalista. Nancy Fraser, en Fortunes of feminism, intenta dar una respuesta a la lógica de Judith Butler: "Capitalism, in other words, 'needs' or benefits from compulsory heterosexuality. It follows, according to Butler, that gay and lesbian

\footnotetext{
${ }^{3}$ Entrevista realizada a la autora por Cápsula de Artes Visuales en 2015. Recuperado de la web oficial de Gabriela Rivera: http://gabrielarivera.blogspot.com/p/portafolio.html.
} 
RAYO CATALINA - Género performativo y crítica de una estructura capitalista: la obra de Gabriela Rivera, Bestiario (2012-2018)

struggles against heterosexism threaten the 'workability' of the capitalist system" (182). Si bien Fraser no está de acuerdo con tal aseveración, pues no considera que el capitalismo esté amenazado por las relaciones no heterosexuales, por mi parte considero que la postura de Butler sí tiene relevancia al momento de pensar en la reproductibilidad humana a favor de un sistema capitalista, especialmente en la obra de Rivera. Por ejemplo, muchos de los nombres de las máscaras reafirman la postura de Butler en Fraser. Yegua, zorra y perra son nombres que fácilmente pueden asociarse a una sexualidad negativa que apela a una supuesta condición biológica heterosexual femenina destinada a la reproducción. La connotación "perra" alude a la hembra animal que, cada vez que está en celo, es seguida por un montón de machos con el propósito de inseminarla.

Ahora bien, el análisis de la obra no solo apela a los términos de reproductibilidad, sino a la lógica capitalista. Si la carne es consumida para alimentarnos, se necesita elevar la producción de esta. Al mismo tiempo, dado que el capital separa estructuras económicas y sociales en una división de esferas binarias: producción/reproducción, público/privado, casa/trabajo, en las que la mujer es relegada a tareas del hogar/casa/lo privado, es de suponer que la tarea de reproducción le es otorgada a ella. Esto se transforma en un círculo de retroalimentación, por lo que la manera de producción económica que maneja el capital termina por re-producir con el propósito de consumir. Se necesita tanto a humanos como animales para su consumo. De ahí que sea posible comprender que el funcionamiento del orden discursivo no solo constituye el imaginario del sexo/género, sino toda una estructura social que permea en el acto de resistencia. David Staples, en su libro No place like home, apunta hacia esta dirección cuando relaciona las tareas 'femeninas' con los objetivos del capital: "homeworking has become tightly interwoven with national and international economic policies, including local and national micro-economic development strategies"5 (2). La obra expresa, así, una ideología opresiva, que no funciona dentro de una lógica conceptual aislada, sino que está entrelazada con la lógica del capital, lo que crea nuevas instancias de opresión hacia lo que significa el cuerpo de la mujer. Carol J. Adams, en The sexual politics of meat: A feminist-vegetarian critical theory, presenta una analogía sobre la violencia ejercida hacia los animales y la violencia de género que padecen las mujeres a partir de la existencia de una cosificación y sexualización respecto de su consumo y reproducción (23).

Las autoras del manifiesto Feminism for the 99 percent, Cinzia Arruzza, Tithi Bhattacharya y Nancy Fraser, explican cómo los métodos de producción y ganancia han instaurado nuevos mecanismos para el entendimiento del cuerpo de la mujer en las estructuras del poder institucional:

But capitalism established new, distinctively "modern" forms of sexism, underpinned

by new institutional structures. Its key move was to separate the making of people

from the making of profit, to assign the first job to women, and to subordinate it to

\footnotetext{
4 "El capitalismo, en otras palabras, "necesita" o se beneficia con la heterosexualidad obligatoria. De ello se desprende, según Butler, que las luchas de gays y lesbianas contra el heterosexismo amenazan la 'viabilidad' del sistema capitalista" (traducido por mí).

5 "las tareas del hogar se han entrelazado estrechamente con las políticas económicas nacionales e internacionales, incluidas las estrategias de desarrollo microeconómico locales y nacionales" (traducido por mí).
} 
the second. With this stroke, capitalism simultaneously reinvented women's oppression and turned the whole world upside down ${ }^{6}(21)$.

Efectivamente, Arruzza et al. expresan el modo en que la mujer y la reproducción es un mero medio para acumular ganancias (22). Esta labor de reproducción es no asalariada, margina a la mujer a dicha función y la coloca en una posición de subordinación.

La obra de Rivera, además de hacernos entender esto, apunta a un feminismo ecológico al considerar un aspecto importante de la materialidad de la obra. Rivera, en vez de utilizar la carne de consumo, utiliza sus residuos para crear sus máscaras. Ahora, estos desechos no valen nada en el mercado, son considerados 'basura'. Entonces, ¿cómo evidencia Rivera los sistemas de opresión a través de lo mercantil si la materialidad no tiene valor? En la entrevista de Fotoféminas, la autora explica que no puede conseguir dichos desechos por sí solos. La manera de obtenerlos es comprando el producto para su consumo entero. Por lo tanto, lo que la artista adquiere (y el consumidor de carne habitual) es un producto "necesario", más un producto "innecesario". El residuo de carne, ese producto "innecesario", es relegado del consumo y, por lo tanto, considerado basura. Dentro de las estructuras binarias que el capital elabora, podríamos argumentar que el producto considerado como carne de consumo, también cae dentro de esta categoría y no es casualidad que la artista haya decidido utilizar el residuo por sobre la carne, que es la que posee un valor económico, develando así las políticas de un mercado respecto de la valorización de los cuerpos.

Desde allí es que Silvia Federici, en El patriarcado del salario: Críticas feministas al marxismo, menciona que estas divisiones son expandidas en varios ámbitos en los que el género juega un papel fundamental. Federici explica, en línea con Ariel Salleh, cómo el trabajo, a lo largo de la historia, cobra sentido al dominar la naturaleza y acomodarla a necesidades humanas. Este razón de ser del trabajo y todo producto que salga de él (lo positivo) es atribuido a lo masculino: "el trabajo se describe como el padre, la naturaleza como la madre (Salleh, 1997:7276) y también la tierra es considerada femenina" (62). No es de extrañar, por lo tanto, que Rivera utilice en su obra dichos residuos, pues pertenecen a la simbolización de lo 'femenino', 'lo otro', 'lo natural', lo que no es un producto positivo dentro del capital. La materialidad de la obra representa este binarismo, en un intento por amoldar y apropiarse de este producto creado con fines económicos. Federici argumenta que "[1]as ecofeministas han demostrado que existe una fuerte conexión entre el desdén hacia el trabajo doméstico, la devaluación de la naturaleza y la idealización de todo lo que produce la industria y la tecnología humana" (62) y es este desdén hacia la naturaleza lo que hace que la obra sea grotesca, tanto en relación con la materialidad como con lo que simboliza ${ }^{7}$.

Por otro lado, no son solo las ecofeministas quienes apuntan a este 'desdén' hacia la naturaleza. Judith Butler escribe un capítulo interesante, en su libro Gender trouble, sobre las inscripciones

\footnotetext{
6 "Pero el capitalismo estableció nuevas formas de sexismo distintivamente "modernas", apuntaladas por nuevas estructuras institucionales. Su movimiento clave fue separar la creación de personas de la obtención de ganancias, asignar el primer trabajo a las mujeres y subordinarlo al segundo. Con este golpe, el capitalismo reinventó simultáneamente la opresión de las mujeres y puso el mundo al revés" (traducido por mí).

${ }^{7}$ De hecho, la misma artista confiesa en la entrevista de Foto-féminas que todo el proceso de manejo de la carne fue tortuoso, puesto que ella es vegana.
} 
RAYO CATALINA - Género performativo y crítica de una estructura capitalista: la obra de Gabriela Rivera, Bestiario (2012-2018)

que se les hace al cuerpo, en el que refiere, en línea con Julia Kristeva, lo abyecto, definiéndolo como: "The 'abject' designates that which has been expelled from the body, discharged as excrement, literally rendered 'Other""8 (181). En este punto, podemos argumentar, entonces, que la obra de Rivera, y no solo el residuo de carne que sus máscaras usan, simboliza la opresión del capital y adquiere significancia en 'lo otro', en lo que no es 'masculino', en esta separación binaria expresada en el género o, como Butler dice más adelante, "The construcción of the "notme""9 (181). Con ello apunta a que en el leguaje de las máscaras, "arpía", solo puede ser atribuido a la mujer, pues es "lo otro", "lo abyecto" y "lo grotesco", establecido mediante la materialidad de la obra, que desvincula todo lo 'femenino' como 'lo otro'. El cuerpo se convierte en una especie de receptáculo de todos los deseos suprimidos del orden narrativo heterocisgénero (Butler, Gender 142).

\section{Vestir la carne, transformarse en ella}

Siguiendo esta idea de las inscripciones del cuerpo que Butler postula, el segundo aspecto destacable de la obra tiene que ver con el uso de las máscaras como acto performativo. En la exposición del Centro Cultural Estación Mapocho en 2015, la obra no solo presentó las fotografías de gran formato, sino que también hubo una muestra de un video performance realizado por la $\operatorname{artista}^{10}$. Este consiste en una toma a cámara fija, con fondo negro, en la que la artista está situada de cuclillas-recostada, desnuda, vistiendo la máscara "Zorra", mientras termina de coserla.

Como bien señala Sagrario Aznar, las performances son acciones efímeras (7), en las que rara vez se le da explicaciones al público sobre lo que significa la acción, sino que se le "bombardea con sensaciones" (8). La propuesta de Rivera nos embarca en un recorrido de sensaciones altamente féminas y vulnerables. Su cuerpo desnudo, en cuclillas-recostada, nos empuja a reflexionar en cómo pensar el cuerpo femenino. Lo vulnerable de este, al estar denudo y en una posición sumisa, nos hace recordar un poco el trabajo Violación (1973) de Ana Mendieta. Si bien, la obra de Rivera no es tan explícita y directa como la de Mendieta, ambas remiten a preguntas relacionadas con el cuerpo femenino y la violencia a la cual siempre pareciera estar sometido. Aznar, en línea con Lucy Lippard, señala las características fundamentales del trabajo de Mendieta "como una creadora preocupada desde el principio por la trilogía de la sangre, la violencia y la fertilidad" (62). La performance de Rivera también cumple con estos tres aspectos: carne (sangre), violencia (palabras) y fertilidad (reproducción/capitalismo).

Ahora bien, para proseguir con un análisis más completo de la performance de Rivera, vuelvo a los aspectos de sumisión mencionados, que refuerzan la analogía de "zorra" con "prostituta". Butler, en Gender trouble, comienza su capítulo "Bodily inscriptions, performative subversions" con la pregunta: "Is 'the body' itself shaped by political forces with strategic interests in keeping that body bounded and constituted by the markers of sex?"11 (175). Con ello

\footnotetext{
8 “'Lo 'abyecto' designa lo que ha sido expulsado del cuerpo, descargado como excremento, literalmente convertido en 'Otro"” (traducido por mí).

9 "La construcción del no-yo" (traducido por mí).

${ }^{10}$ La video performance se puede ver en la página web de la artista: http:/gabrielarivera.blogspot.com/p/portafolio.html.

11 “¿'El cuerpo’ en sí mismo es moldeado por fuerzas políticas con intereses estratégicos en mantener ese cuerpo ligado y constituido por las marcas de su sexo?" (traducido por mí).
} 
señala que el cuerpo está determinado políticamente por el sexo. En el caso de la obra de Rivera, ¿sería posible argumentar que estas mismas fuerzas políticas inscriben en el cuerpo la palabra "zorra"? Siguiendo la misma lógica de Butler, podemos argumentar que el cuerpo desnudo de la artista es un medio pasivo al cual se le atribuye una significación que proviene de un entendimiento cultural externo; por ende, estas atribuciones culturales quedan inscritas en el cuerpo. En su texto, Butler habla sobre la problemática de la interpretación del cuerpo, al ser este ubicado en una sociedad. En línea con Foucault, desde lo que este plantea como el deseo, la autora remarca que "the body is the inscribed surface of events" 12 (176). Butler argumenta que los eventos inscritos en el cuerpo son el objetivo de la historia, que destruye y preserva el cuerpo. Lo interesante es que la historia es la creadora de los valores y significados que el cuerpo necesita como sujeto para expresarse (Butler, Gender 177).

Por tanto, ¿qué está pasando con este cuerpo performático de Rivera? Si el cuerpo es un medio pasivo para la inscripción de eventos que otorgan valores y significados entregados por la historia, ¿qué entiende la historia cuando se enfrenta a un cuerpo femenino desnudo? Aquí es importante comprender la 'cotidianeidad' de la palabra. Lo cotidiano es rutinario, un suceso de eventos que se repite con el objetivo de que todos entendamos social y culturalmente el contexto en el cual nos encontramos. Las inscripciones "zorra", "arpía", "mosca muerta" que Rivera apunta son, en este caso, valores que se le han atribuido a un cuerpo específico. El acto de la performance de Rivera, coser constantemente la máscara de zorra (intentando terminarla cuando todavía la viste) es, tal vez, la manera como entendemos que el cuerpo es una construcción social al inscribir la máscara con tantas variantes repletas de significado a través de la historia. Butler, sin embargo, en su artículo "Foucault and the paradox of bodily inscriptions", apunta una crítica a la visión de Foucault al preguntarse si realmente existe "un cuerpo" externo a la construcción cultural que pueda representar la resistencia de la cultura (Butler, Paradox 602). Bajo esta lógica, ¿podemos realmente des-vincular 'lo que significa' ("zorra") de un cuerpo específico (mujer desnuda)?

Cuando la artista se 'auto-impone' la máscara de zorra (es decir, cuando se la sujeta firmemente, como si no quisiera quitársela, incluso para terminar de coserla), se puede vislumbrar también su propio proceso histórico, cómo el cuerpo se somete a estas inscripciones y cómo, a pesar de que la máscara no es parte del cuerpo (sino algo externo), el cuerpo se apropia de ella, 'la hace suya', construyéndola y usándola. Parte de la significación de este acto performático, en cuanto cuerpo que habita bajo un orden de costumbres y construcciones sociales del imaginario colectivo, tiene que ver con el proceso en que la mujer termina creyendo las significaciones que se le han otorgado: "si me visto de cierta manera, la gente puede creer que soy fácil"'.

Este proceso de significación hace que entendamos el cuerpo como sometido o, como Butler dice, un "medio pasivo" (Gender 175), pues no es el cuerpo quien construye los valores y significados sobre sí mismo, simplemente lo expresa a través de lo performativo:

In other words, acts and gestures, articulated and enacted desires create the illusion of an interior and organized gender core, an illusion discursively maintained for the

\footnotetext{
12 "el cuerpo es la superficie inscrita de los eventos" (traducido por mí).
} 
RAYO CATALINA - Género performativo y crítica de una estructura capitalista: la obra de Gabriela Rivera, Bestiario (2012-2018)

purpose of the regulation of sexuality within the obligatory frame of reproductive heterosexuality ${ }^{13}$ (Butler, Gender 185).

Butler argumenta que los actos y gestos que el cuerpo expresa son solo para mantener un discurso heterocisgénero con propósitos reproductivos, pero que no nacen 'naturalmente' a partir del cuerpo. Es decir, no por ser mujer, 'naturalmente seré femenina', sino que, al fin y al cabo, se trata de actos performáticos para mantener una heterosexualidad obligatoria en un sistema que perpetúa la violencia de género, un sistema reforzado por el capital: reproducirse para consumir.

Si nuestros actos performáticos no nacen naturalmente a partir de nuestro interior, ¿de dónde provienen? Cuando Butler critica la postura de Foucault, subraya un aspecto interesante: las inscripciones marcadas en nuestros cuerpos implican un poder fuera de este: "Although Foucault appears to argue that the body does not exist outside the terms of its cultural inscription, it seems that the very mechanism of 'inscription' implies a power that is necessarily external to the body itself"14 (Butler, Paradox 603). Esto significa que el cuerpo es sumiso a este poder que está fuera de su control. ¿Podemos entonces argumentar que el problema cultural no recae en el cuerpo, sino en la historia que crea estas inscripciones en nosotros?

\section{Conclusión: la palabra se viste y se queda impregnada en el cuerpo}

La obra de Rivera expresa distintos problemas de significancia en el acontecer actual de Chile, desde problemáticas relacionadas con el capital, hoy neoliberalismo, como aquellas vinculadas con lo performático del género. Ambas esferas expresan, en la obra de Rivera, una violencia de género a través de la opresión capitalista, como un tema socio-cultural-histórico. Valentina Saavedra y Javiera Toro plantean que la violencia tiende a ser relacionada con la violencia más directa, la que es más fácil de ver. Las autoras del artículo "La revuelta feminista: de la lucha de las mujeres a la lucha por una nueva sociedad" hablan de cómo en un país como Chile pueden existir 47 femicidios anuales pero, "como dicen las feministas, esa es la punta de iceberg de un entramado de violencia física, sicológica y económica que construye una estructura de la relación social de dominación entre hombres y mujeres" (138).

Cuando la obra de Rivera apunta a la violencia de género, no lo hace a partir de una violencia explícita, como un feminicidio (o como algunas obras de Ana Mendieta), sino que pone en escena una violencia sistemática-rutinaria traducida en el lenguaje. Por ejemplo, al hacer un registro de video de su performance -en contra de una de las características principales de las performances que, como indica Aznar, es su particularidad efímera (igual que la palabra al aire)hace que la acción representada se reproduzca infinitamente, transformándola en rutina (en lo cotidiano). Por tanto, mientras que a nuestros cuerpos femeninos se les somete las inscripciones de "zorra", "arpía", "yegua", "perra", "víbora", "mosca muerta", "pájara", "cerda", de forma

\footnotetext{
13 “En otras palabras, los actos y gestos, los deseos articulados y promulgados crean la ilusión de un núcleo de género interior y organizado, una ilusión discursivamente mantenida con el propósito de regular la sexualidad dentro del marco obligatorio de la heterosexualidad reproductiva" (traducido por mí).

14 "Aunque Foucault parece argumentar que el cuerpo no existe fuera de los términos de su inscripción cultural, parece que el mecanismo mismo de "inscripción' implica un poder que es necesariamente externo al cuerpo mismo" (traducido por mí).
} 
rutinaria y cotidiana, la violencia expuesta es que, a través del tiempo, se pueden ir agregando más inscripciones como estas, que no se destruyen, y si lo hacen, son para crear nuevas inscripciones.

Iris Marion Young distingue asertivamente la relación entre lo que se vive en el cuerpo y el género. En congruencia con las ideas de Butler, Young afirma que desde una edad muy temprana nuestros cuerpos están sometidos a aspectos culturales (inscripciones):

The body as lived is always enculturated: by the phonemes a body learns to pronounce at a very early age, by the clothes the person wears that mark her nation, her age, her occupational status, and in what is culturally expected or required of women $^{15}(17)$.

Young no solo se queda en la afirmación de las expectativas culturales, sino que plantea que es el mismo cuerpo quien reacciona frente a dichas expectativas: "the person experiences herself as looked at in certain ways, described in her physical being in certain ways, she experiences the bodily reactions of others to her, and she reacts to them"16 (17). Esto esclarece cómo nuestros cuerpos reaccionan frente a estas inscripciones. En Bestiarios, lo que Rivera intenta expresar es que adoptamos estas inscripciones culturales de la palabra y nos las cocemos en nuestros cuerpos, como si fuera un ritual que se repite infinitamente. En el caso de la artista, un ritual de la costura que nace desde el núcleo familiar.

Aquí, una de las cosas que Kirkwood hábilmente apunta es la importancia de la tradición:

[...] lo que significa que el origen del dominio esta siempre en una comunidad familiar, con relaciones de parentesco jerarquizadas. Ahora bien, para Weber, la legitimidad de esta autoridad radica en la tradición, esto es: "en la creencia en el carácter inquebrantable de lo que ha sido siempre de una manera determinada" (49).

Por lo tanto, cabe preguntarse, al momento de entender la performance de Rivera, si las inscripciones en nuestros cuerpos son 'enseñadas' por nuestro núcleo familiar. Y también cómo es posible traspasar estas enseñanzas. Quizás, volver la mirada hacia los escritos de Kirkwood

15 "El cuerpo vivido siempre está inculturado: por los fonemas que un cuerpo aprende a pronunciar a una edad muy temprana, por la ropa que lleva la persona que marca su nación, su edad, su situación ocupacional y lo que culturalmente se espera o exige de la mujer" (traducido por mí).

16 "la persona se experimenta a sí misma cuando se la mira de ciertas maneras, descrita en su ser físico de ciertas maneras, experimenta las reacciones corporales de los demás hacia ella y reacciona ante ellas" (traducido por mí). 
RAYO CATALINA - Género performativo y crítica de una estructura capitalista: la obra de Gabriela Rivera, Bestiario (2012-2018)

tiene más sentido de lo que podríamos creer. La revolución feminista ha ayudado a conformar un movimiento contemporáneo basado en la rebeldía, en la mirada de la mujer, en 'lo otro', en lo abyecto y esto se puede ver en la producción artística local. 


\section{Bibliografía}

Adams, Carol J. The sexual politics of meat: A feminist-vegetarian critical theory. Londres: Bloomsburry Revelations, 2015. Impreso.

Arruzza, Cinzia, Tithi Bhattacharya y Nancy Fraser. Feminism for the 99 percent: A manifesto. Londres: Verso, 2019. Impreso.

Aznar, Sagrario. El arte de acción. San Sebastián: Nerea, 2006. Impreso.

Butler, Judith. "Foucault and the paradox of bodily inscriptions". The Journal of Philosophy, 86.11, Eighty-Sixth Annual Meeting American Philosophical Association, Eastern Division (1989): 601-607.

Mecanismos psíquicos del poder: Teorías sobre la sujeción. Madrid: Cátedra, 2001. Impreso.

-------- Gender trouble. Londres: Routledge, 2006 [1990]. Impreso.

Castillo, Alejandra. Julieta Kirkwood: Políticas del nombre propio. Santiago: Palinodia, 2007.

------- "De la revuelta feminista, la historia y Julieta Kirkwood". Mayo feminista: La rebelión contra el patriarcado. Faride Zerán (ed.). Santiago: LOM, 2018. Impreso.

Federici, Sylvia. El patriarcado del salario: Críticas feministas al marxismo. María Aránzazu Catalán Altuna, Carlos Fernández Guervós y Paula Martín Ponz (traductores). Buenos Aires: Tinta Limón, 2018. Impreso.

Fraser, Nancy. "Heterosexism, misrecognition, and capitalism: A response to Judith Butler". Fortunes of feminism: From state-managed . Londres: Verso, 2013. Impreso.

Kirkwood, Julieta. Feminarios. San Antonio: Documentas, 1987. Impreso.

Pinto, Patricia. "Un pensamiento alternativo en el ensayo latinoamericano: 'Feminarios', de Julieta Kirkwood”. Chasqui, 21.1 (1992): 55-66.

Saavedra, Valentina y Javiera Toro. "La revuelta feminista: de la lucha de las mujeres a la lucha por una nueva sociedad". Mayo feminista: La rebelión contra el patriarcado. Faride Zerán (ed.). Santiago: LOM, 2018. Impreso.

Salinero, Mónica y Stella Salinero. "Cría cuervas, exposición de Gabriela Rivera". Atlas, Revista Fotografía e Imagen (4.1.2016). Web. https://atlasiv.com/2016/01/04/cria-cuervasexposicion-de-gabriela-rivera/.

Valdés, Ximena. "Una fiesta otoñal: Notas sobre la emergencia de la nueva ola feminista". Mayo feminista: La rebelión contra el patriarcado. Faride Zerán (ed.). Santiago: LOM, 2018. Impreso.

Staples, David. No place like home: Organizing home-based labor in the era of structural adjustment. Londres: Routledge, 2007. Impreso.

Young, Iris Marion. On female body experiences: "Throwing like a girl” and other essays. Oxford: Oxford University Press, 2005. Impreso. 